\begin{tabular}{c|c|c}
$\substack{\text { Teoría de la Educación } \\
\text { ENiveración y Cultura en la } \\
\text { BSALAMANCA }}$ & Sociedad de la Información \\
\hline TESI, 15(3), 2014, pp. 1-255 &
\end{tabular}

\title{
DESARROLLO DE APRENDIZAJES ACTIVOS EN PRIMEROS CURSOS UNIVERSITARIOS: WORKSHOP CÓNICAS
}

\begin{tabular}{|c|c|c|c|}
\hline M. Carmen & M. Luisa & José FÁBREGA & Luis \\
\hline MORILLO & CASADO & GOLPE & SEBASTIÁN \\
\hline BALSERA & FUENTE & Doctor Ingeniero de Caminos & LORENTE \\
\hline $\begin{array}{l}\text { Doctora en Matemáticas. } \\
\text { ETSI Topografía, Geodesia y } \\
\text { Cartografía. Universidad } \\
\text { Politécnica de Madrid } \\
\text { (UPM). Departamento de } \\
\text { Ingeniería Topográfica y } \\
\text { Cartografía. } \\
\text { mariadelcarmen.morillo@upm }\end{array}$ & $\begin{array}{l}\text { Licenciada en Matemáticas } \\
\text { ETSI Topografía, Geodesia y } \\
\text { Cartografía. Universidad } \\
\text { Politécnica de Madrid } \\
\text { (UPM). Departamento de } \\
\text { Ingeniería Topográfica y } \\
\text { Cartografía. } \\
\text { ml.casado@upm.es }\end{array}$ & $\begin{array}{l}\text { ETSI Topografía, Geodesia y } \\
\text { Cartografía. Universidad } \\
\text { Politécnica de Madrid } \\
\text { (UPM). Departamento de } \\
\text { Ingeniería Topográfica y } \\
\text { Cartografía. } \\
\text { jose.fabrega@upm.es }\end{array}$ & $\begin{array}{l}\text { Licenciado en Matemáticas } \\
\text { ETSI Topografía, Geodesia y } \\
\text { Cartografía. Universidad } \\
\text { Politécnica de Madrid } \\
\text { (UPM). Departamento de } \\
\text { Ingeniería Topográfica y } \\
\text { Cartografía. } \\
\text { luis.sebastian@upm.es }\end{array}$ \\
\hline
\end{tabular}

Resumen:

Como ya es conocido, los profesores de Matemáticas utilizamos los ejemplos como recursos de aprendizaje para enseñar algún contenido matemático concreto, de modo que las generalizaciones y abstracciones sean más fácilmente entendidas por los alumnos, pasando de lo concreto a lo abstracto, como otra forma de enseñar y practicar en Matemáticas. Esta metodología de trabajo se ve potenciada por el uso de dispositivos móviles llamados mobile-learning (m-learning) o educación móvil (educación-m), en español. Siguiendo esta línea de trabajo, se ha realizado el workshop de cónicas que se presenta en este artículo, empleando estas nuevas tecnologías (TIC) y con el objetivo de desarrollar aprendizajes activos en Geometría a través de la resolución de problemas en los primeros cursos de Grado en las ingenierías.

Palabras clave: workshop, aprendizaje, TIC, Geometría.

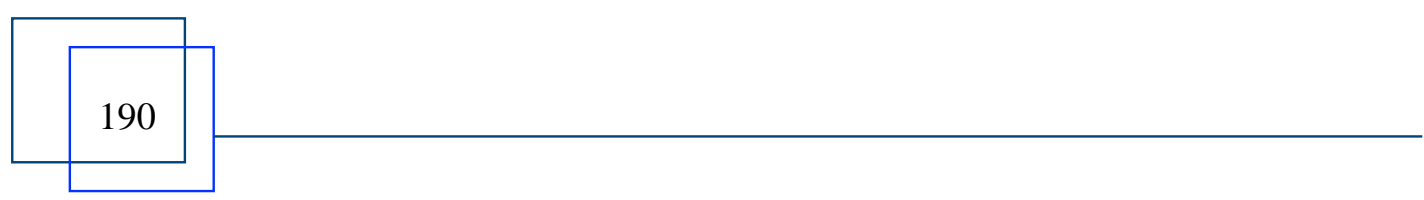




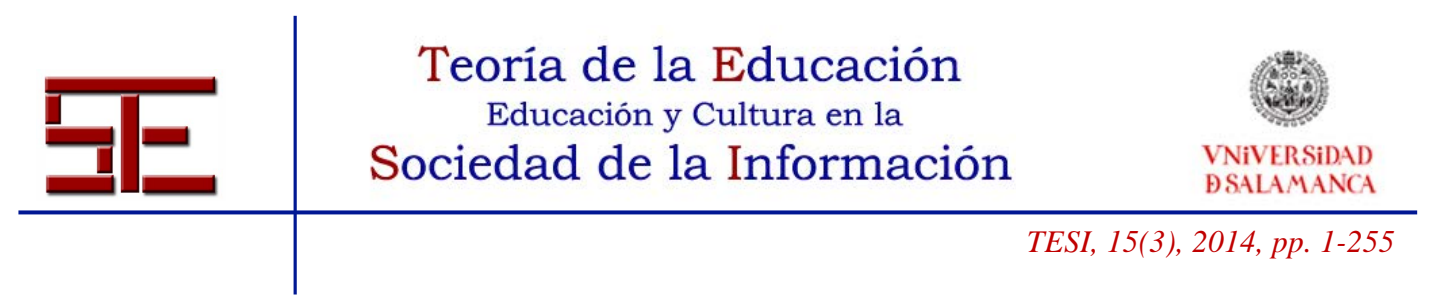

\title{
ACTIVE LEARNING DEVELOPMENT AT UNIVERSITY FIRST YEARS: WORKSHOP OF CONIC SECTIONS.
}

\begin{abstract}
:
As it is already known, math teachers, use examples as learning resources, to teach some specific math contents, so that generalizations and abstractions are more easily understood by students, from concrete to abstract, as another way of Mathematics teaching and training. This methodology is enhanced by the use of mobile devices, called mobile-learning (m-learning) o "educación móvil" (educación-m), in Spanish. Following this strategy, the workshop of conic sections shown in this paper has been carried out, using these new technologies (ICT) and in order to develop active learning in Geometry through problem-solving at the first years of engineering degrees.
\end{abstract}

Key words: workshop, learning, ICT, Geometry. 


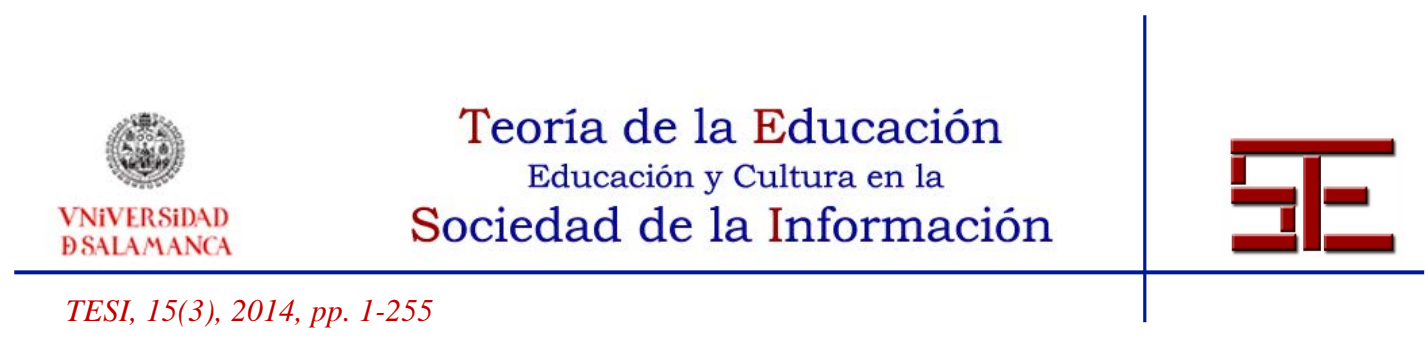

\section{INTRODUCCIÓN}

El aprendizaje y la metodología de enseñanza de las Matemáticas con las nuevas tecnologías, ha creado un nuevo tipo de enseñanza para lograr la efectividad del proceso de aprendizaje. En el artículo "Las nuevas tecnologías y el aprendizaje de las matemáticas", se comenta: "Es evidente el acelerado desarrollo de la tecnología, se ha demostrado que en los últimos cincuenta años se han presentado las más revolucionarias innovaciones, y que inevitablemente toca y afecta a la sociedad en su conjunto. El lenguaje y la comunicación no han escapado de los cambios, ya desde la antigüedad, la imprenta es causal de un cambio radical en el lenguaje escrito y ahora éste, ha revolucionado con la era de la electrónica y la computación; surgen las llamadas nuevas tecnologías de la comunicación y la información (TIC) y por extensión los medios informáticos, y su principal instrumento: la computadora" (Macias, 2007, 1), lo cual constituye un resumen de lo que realmente ha ocurrido.

Además, utilizar las TIC en la enseñanza puede proporcionar mayor motivación de aprender por parte del alumno. De tal forma que adquiere gran importancia la reflexión de Cabero y Llorente cuando afirma que: "En líneas generales podemos decir que las nuevas tecnologías se apoyan en las telecomunicaciones, la informática y los audiovisuales y su hibridación como son los multimedia; y lo hacen no de forma individual sino interactiva e interconexionada, lo que permite conseguir nuevas realidades comunicativas y potenciar las que pueden tener de forma aislada" (Cabero, 2000, 191).

Con los avances tecnológicos señalados anteriormente, si además consideramos la evolución de las redes inalámbricas, es fácil entender cómo los móviles han cobrado enorme importancia en la educación. Es una modalidad educativa que facilita la construcción del conocimiento, la resolución de problemas de aprendizaje y el desarrollo de destrezas, es el mobile learning (m-learning). El mobile learning está implícito en muchas de las teorías educativas: teoría de la actividad, teoría del conectivismo, teoría del conductismo, teoría del constructivismo social, aprendizaje colaborativo y aprendizaje informal.

Como ejemplo de la importancia que actualmente viene dándose al m-learnig, citaremos el blog del Centro de Innovación e Investigación Educativa (cniie), en la Semana del Aprendizaje Móvil (21 de febrero, 2014) en la conferencia emblemática de la UNESCO sobre las tecnologías móviles y la educación.

Por otra parte, innovar en educación es buscar otro tipo de aprendizaje que complemente a los que ya existen, con el objetivo de hacerlo más eficaz. Según Aguaded Gómez: "Referirse a innovación en la educación supone tener presente una pluralidad de conceptos y perspectivas teóricas e ideológicas que permiten comprender, analizar y explicar las decisiones que buscan cambiar y transformar la escuela"

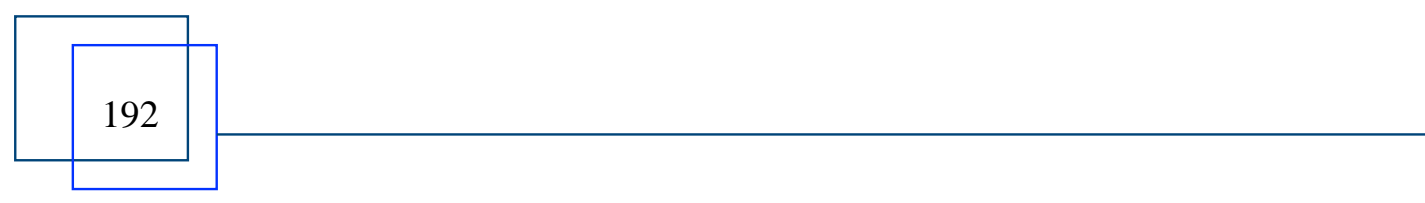




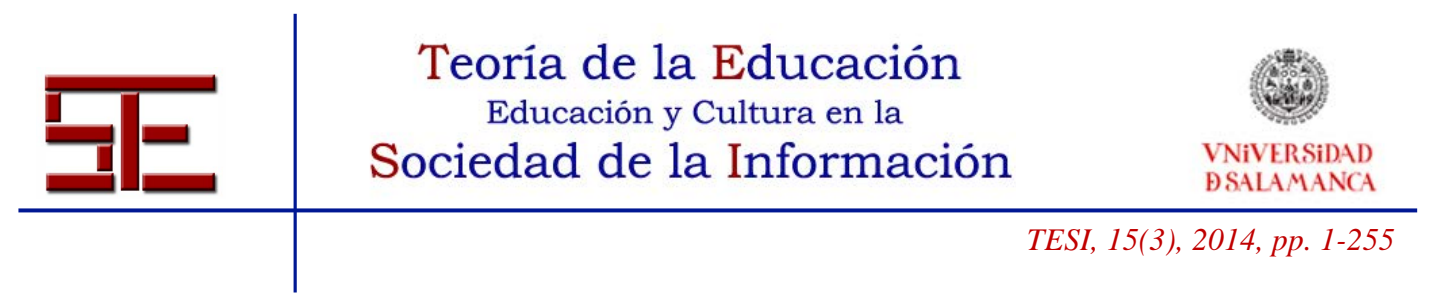

(Aguaded, 2010, 1).

Ante este panorama, el reto a futuro está en que las Universidades innoven no solo su tecnología, sino también sus concepciones y prácticas pedagógicas, lo que significa modificar el modelo de enseñanza universitario en su globalidad. En el terreno de la Educación Superior existe una gran cantidad de literatura en torno al tema, que refleja la preocupación constante por la actualización y la discusión en torno a las características de la formación y el perfil profesional del egresado de la universidad del futuro (Wikilibros, 2009).

Este artículo propone aplicar todo lo anteriormente dicho a una temática específica de la Geometría, donde los profesores ofrecemos una nueva forma de enseñar, informar, compartir y entusiasmar. En este caso concreto, lo hemos aplicado al tema de cónicas, donde especialmente hemos desarrollado unos talleres virtuales formados por materiales didácticos y un proceso de autoevaluación por parte del alumno. Para la realización de los talleres se han utilizado recursos de las nuevas tecnologías de la información y las comunicaciones. Recordamos a Barrows en su definición del aprendizaje cuando dice "un método de aprendizaje basado en el principio de usar problemas como punto de partida para la adquisición e integración de los nuevos conocimientos" (Barrows, 1986, 481) y desde el punto de vista de Prieto cuando señala que "el aprendizaje basado en problemas representa una estrategia eficaz y flexible que, a partir de lo que hacen los estudiantes, puede mejorar la calidad de su aprendizaje universitario en aspectos muy diversos" (Prieto, 2006, 173); en estas dos observaciones está la esencia de la metodología de la investigación que hemos aplicado.

\section{CONTEXTO}

El matemático griego Menecmo (sobre el 350 a. C.) incorporó estas curvas al saber conocido de su época y fue Apolonio de Perga (262-190 a. C.) quien clasificó las cónicas en tres tipos: elipses, hipérbolas y parábolas.

Esto fue el comienzo.

Actualmente las curvas de segundo grado son aplicadas a importantísimos fenómenos estudiados en la naturaleza, en ingeniería, arquitectura,..., es decir, las aplicaciones de las cónicas son abundantes. El profesor físico-matemático Vagn Lundsgaard Hansen expone: "Por ejemplo, las propiedades de reflexión de la elipse son aprovechadas en la destrucción de los cálculos renales y también las de la parábola en las antenas parabólicas. Para realizar ciertos movimientos mecánicos en los robots se necesitan engranajes elípticos. La hipérbola es aprovechada en navegación (navegación hiperbólica, sistemas Navegadores Decca). Sin apenas darnos cuenta, de muchas maneras las secciones cónicas son parte de nuestra vida diaria" (Hansen, traducción de

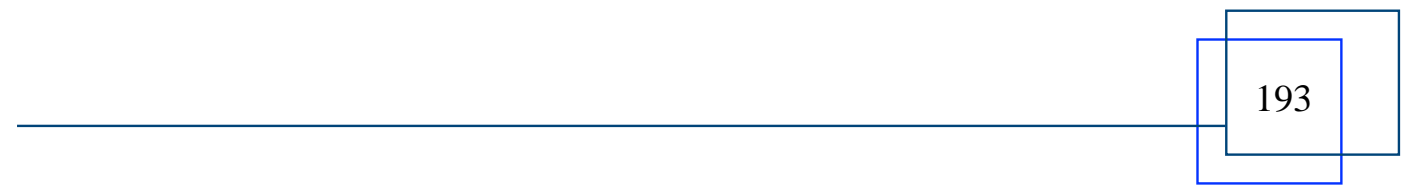




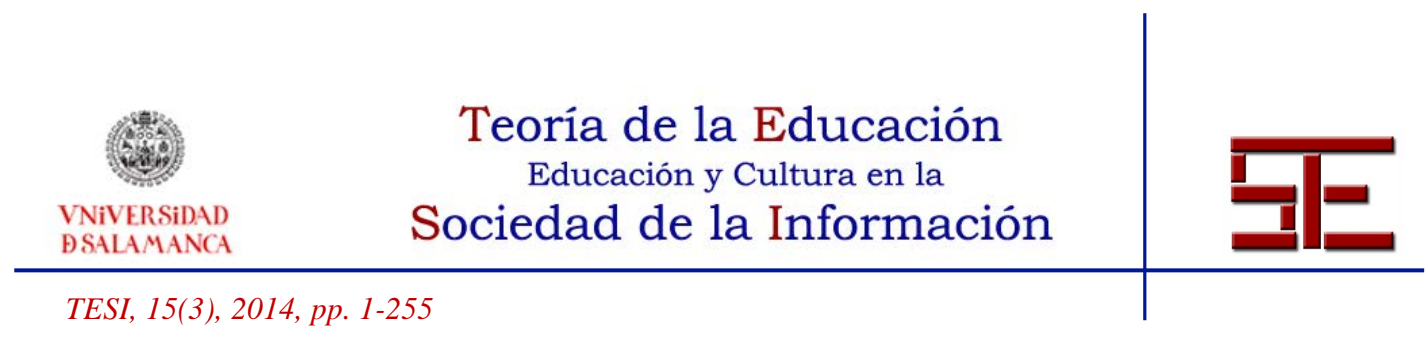

Hernández, V., Villalba M., 2001, 2).

El físico-matemático J. L. Synge escribió: “Además de las rectas, círculos, planos y esferas que conoce cualquier estudiante de Euclides, los griegos sabían las propiedades de las curvas que se obtienen al cortar un cono con un plano: la elipse, la parábola y la hipérbola. Kepler descubrió al analizar sus observaciones astronómicas -y Newton lo demostró matemáticamente sobre la base de la ley universal de la gravitación- que los planetas describen elipses. Así se hizo de la geometría de la Grecia antigua piedra angular de la astronomía moderna" (Synge, 1959, 257).

Es nuestro propósito contribuir a mejorar la enseñanza de estas curvas de segundo grado, mediante las TICs. Su estudio comienza al final del bachillerato. Los conceptos relacionados con ellas como excentricidad, ejes, focos, vértices, los conoce el alumno que accede a la Universidad.

Sin embargo, las secciones cónicas aparecen en algunos aspectos de las ciencias y las ingenierías $\mathrm{y}$, en este caso, tienen la peculiaridad de que los ejes de dichas secciones cónicas no son paralelos a los ejes coordenados. Este es el caso de las elipses de error en la medida de coordenadas o los acuerdos parabólicos en el perfil longitudinal del proyecto de las obras de carreteras y autopistas.

En este caso la cónica se presenta en su ecuación general:

$$
a x^{2}+b x y+c y^{2}+d x+e y+f=0
$$

Siendo preciso clasificarla y obtener sus elementos característicos.

Asimismo, es importante que, dado que es parte fundamental del proceso, el alumno debe saber analizar la matriz resultante de la ecuación $\mathrm{X}^{\mathrm{t}} \mathrm{AX}=0$ y entienda el concepto de valor propio de una matriz y su relación con las direcciones de los ejes principales de las cónicas. Todo esto ha sido el objetivo de este trabajo en el workshop de cónicas que exponemos.

\section{DESCRIPCIÓN}

Para la realización del trabajo, en primer lugar nos preguntamos qué conocimientos de partida poseían los alumnos y cuál es el objetivo a alcanzar. Ausbel, afirma: "Si tuviese que reducir toda la psicología educativa a un solo principio, enunciaría este: El factor más importante que influye en el aprendizaje es lo que el alumno ya sabe. Averígüese esto y enséñese consecuentemente" (Ausbel, 1976, 6).

Teniendo en cuenta esta reflexión, el proyecto consistió en diseñar y describir los flujos de trabajo para realizar el proceso completo, desde la introducción de la ecuación de la cónica a su clasificación y obtención de todos sus elementos. Para ello se dispuso de toda la base teórica en forma de documentos accesibles por el alumno. Este workshop se

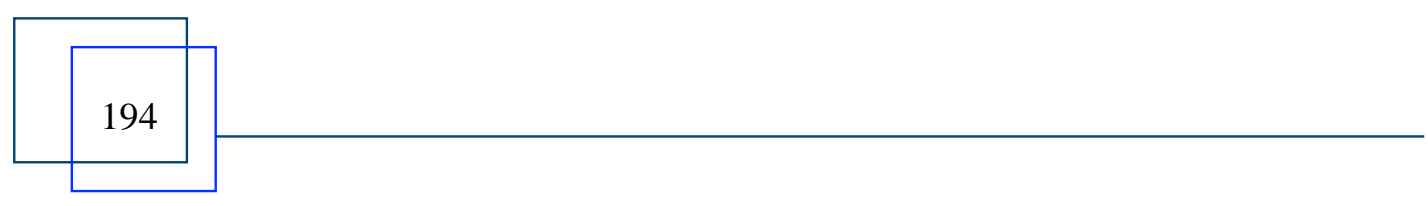




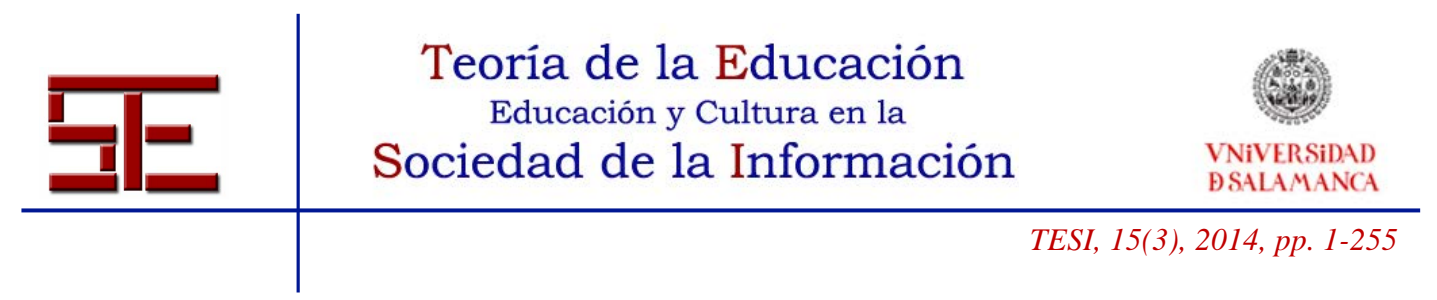

apoya, fundamentalmente, en la plataforma Moodle, que se viene utilizando hace varios años en la Universidad Politécnica de Madrid. Este flujo de trabajo se ha descrito en detalle para realizarlo a mano $o$ bien con el programa Wolfram (<http://www.wolframalpha.com/>), programa que proporciona respuestas y visualizaciones inmediatas; disponibles en un teléfono móvil. Constituye la versión gratuita del programa MATHEMATICA.

Se grabaron unos videos explicativos en los cuales el profesor va realizando "paso a paso" el proceso y proponiendo una serie de problemas resueltos para que el alumno pueda afirmar sus conocimientos resolviendo cualquier tipo de cónica que se le presente.

La utilización del programa Wolfram nos permitió ver la representación gráfica de las cónicas y sabemos que la visualización juega un papel muy importante en la enseñanza de las matemáticas. Además, es una tarea del docente de matemáticas: la enseñanza de la visualización, que va más allá de educar en el conocimiento de la estructura formal y lógica de cualquiera de sus campos, tal y como señala Miguel de Guzmán (1996).

También se han realizado una serie de test de autoevaluación para que el alumno compruebe sus progresos en el taller.

\subsection{Descripción detallada del WorkShop de cónicas}

El WorkShop de Cónicas se encuentra en la siguiente dirección web: <http://moodle.topografia.upm.es/course/view.php?id=45>.

Consta de cinco bloques (ver Fig. 1). 

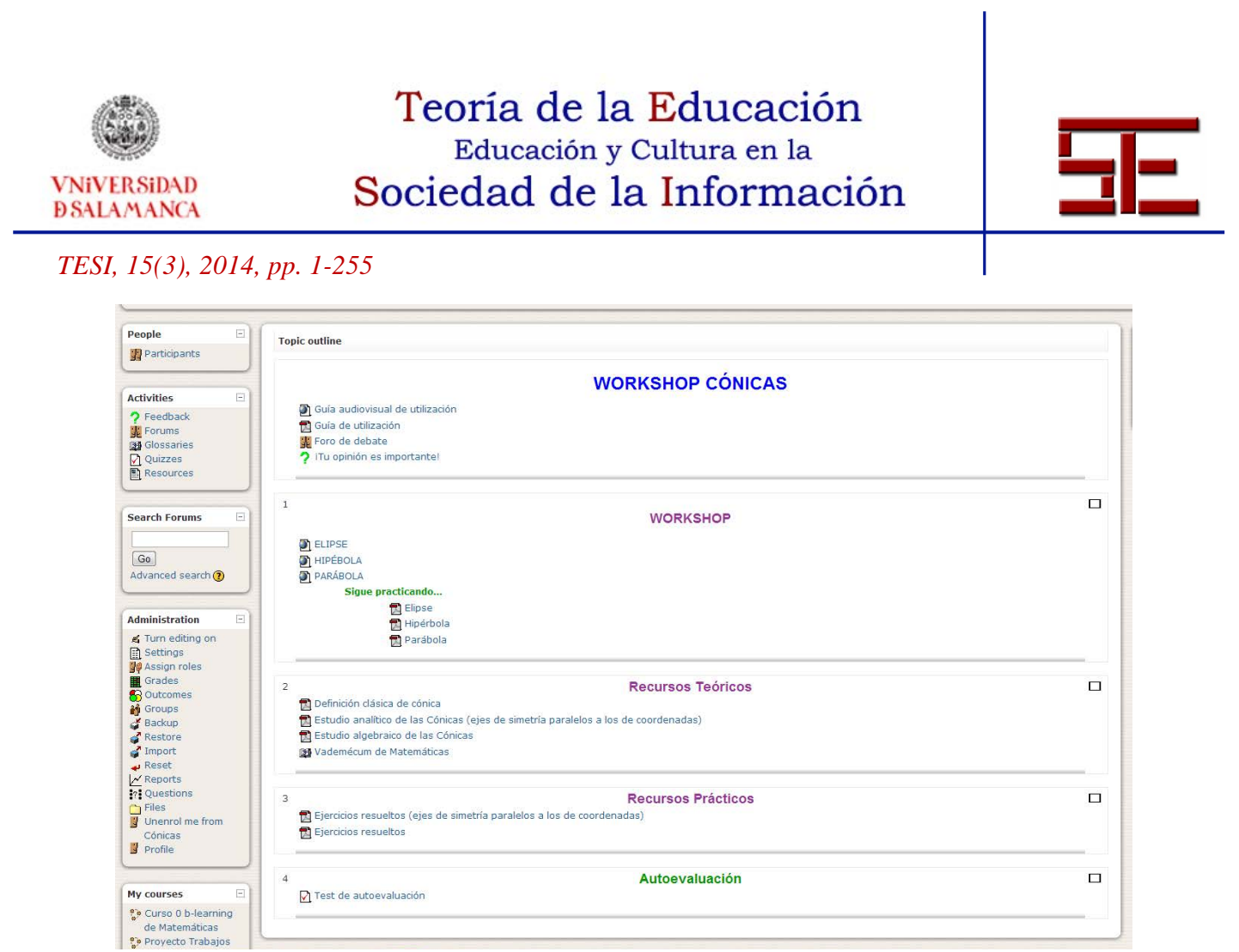

Fig. 1. Presentación de WorkShop Cónicas.

Estos bloques son:

1. Presentación de WorkShop Cónicas. Se presenta un video de dicho taller, indicando la metodología llevada a cabo así como el material disponible y el perfil del alumnado. Además, en este bloque se encuentra un PDF resumen del video de presentación, un foro de debate y un apartado al que se ha llamado “¡Tu opinión es importante!”, el cual es una encuesta cuya finalidad es ir mejorando el WorkShop.

2. Workshop. Este bloque es el núcleo fundamental, ya que en él se encuentran los videos explicativos de los diferentes tipos de cónicas, además de proponer en PDF problemas muy relacionados con los videos anteriormente mencionados con el objetivo de fortalecer el conocimiento adquirido. Estos archivos tienen marcadores (a modo de índice) y haciendo "clic" en cada uno de los apartados aparece la solución correspondiente (ver Fig. 2). 


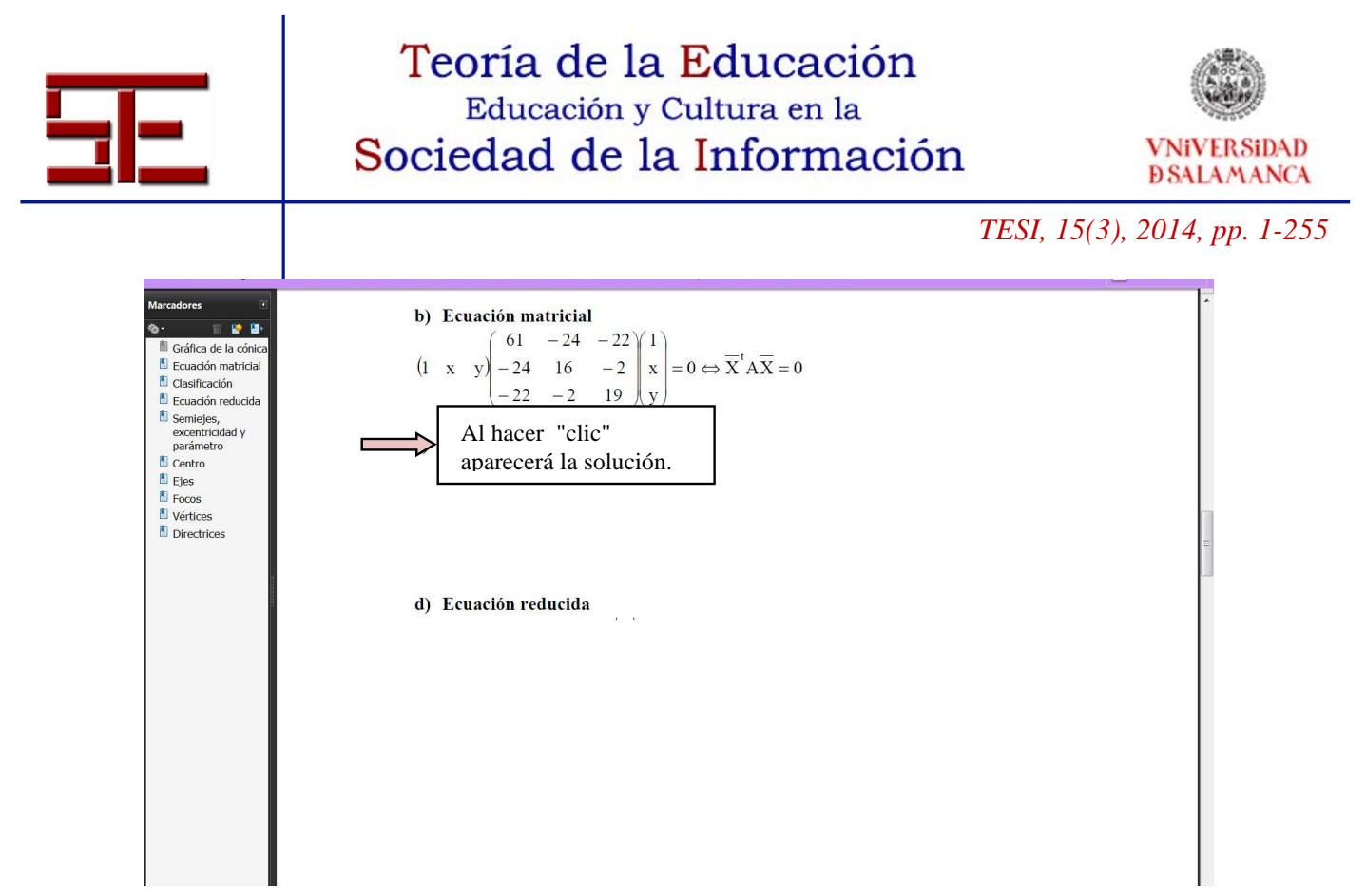

Fig. 2. Archivo de "Sigue practicando".

3. Recursos teóricos: hay tres archivos, el primero para recordar la definición geométrica clásica de cónica, el segundo es la teoría correspondiente al estudio analítico de las cónicas y el tercero es el estudio algebraico de las mismas. Hay que destacar que estos archivos tienen marcadores, lo que permite ir directamente al concepto que se quiera recordar o estudiar (ver Fig. 3).

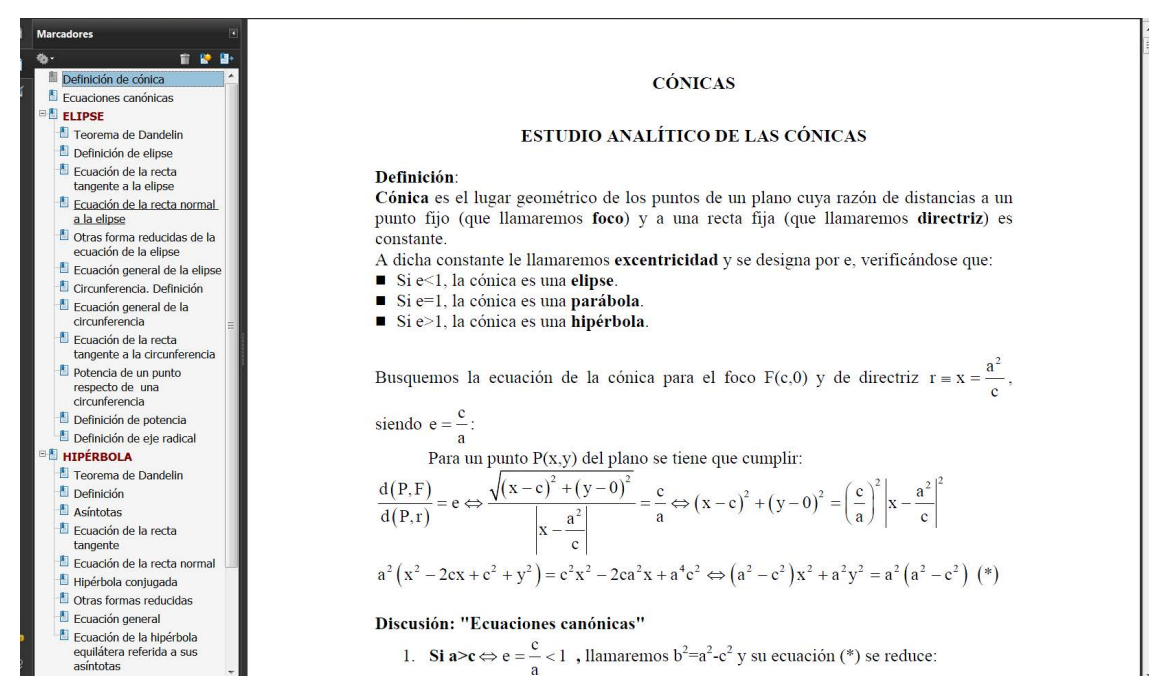

Fig. 3. Archivo de "Estudio analítico de las Cónicas". 


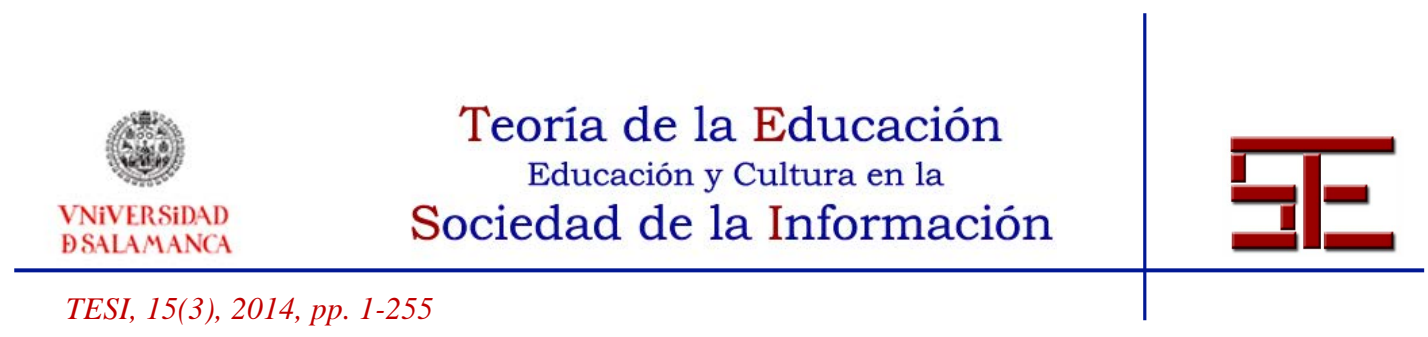

En este bloque hay que subrayar el Vademécum, glosario de conceptos matemáticos que recoge 770 entradas (definiciones, fórmulas y teoremas). Al marcar "Search full text", se muestran todos los conceptos donde aparece la palabra que se busca. Si no se tiene marcada esta opción, solo nos mostrará la definición del concepto pedido (ver Fig. 4).

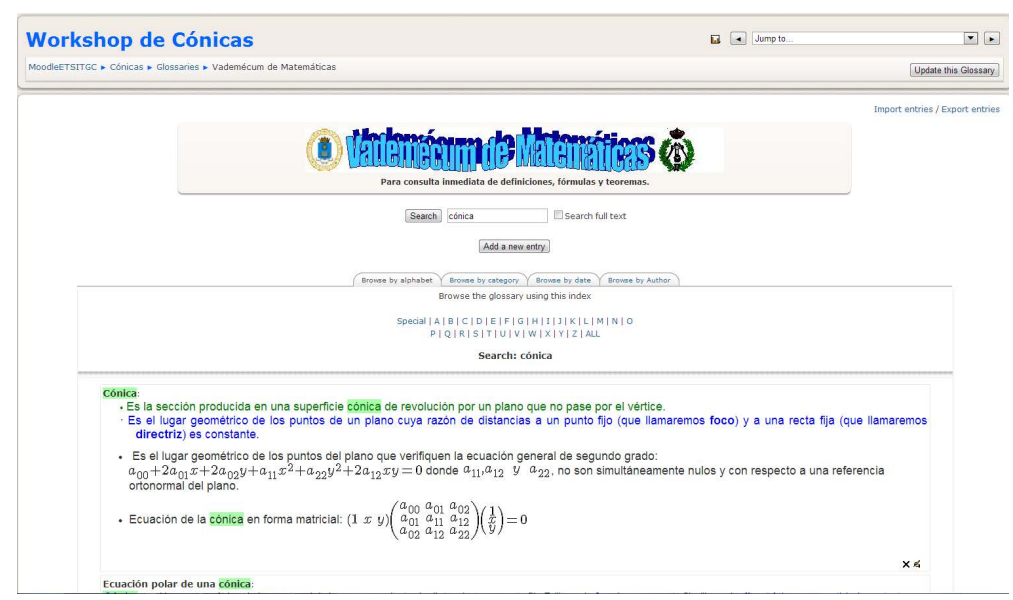

Fig. 4. Vademécum de Matemáticas.

4. Recursos prácticos, consiste en dos archivos con diferentes tipos de problemas, todos resueltos. Los archivos tienen marcadores con el objetivo de que el alumno pueda ir directamente al tipo de problema que busca y a continuación, si quiere saber la solución, no tiene más que pulsar "Solución" (ver Fig. 5).

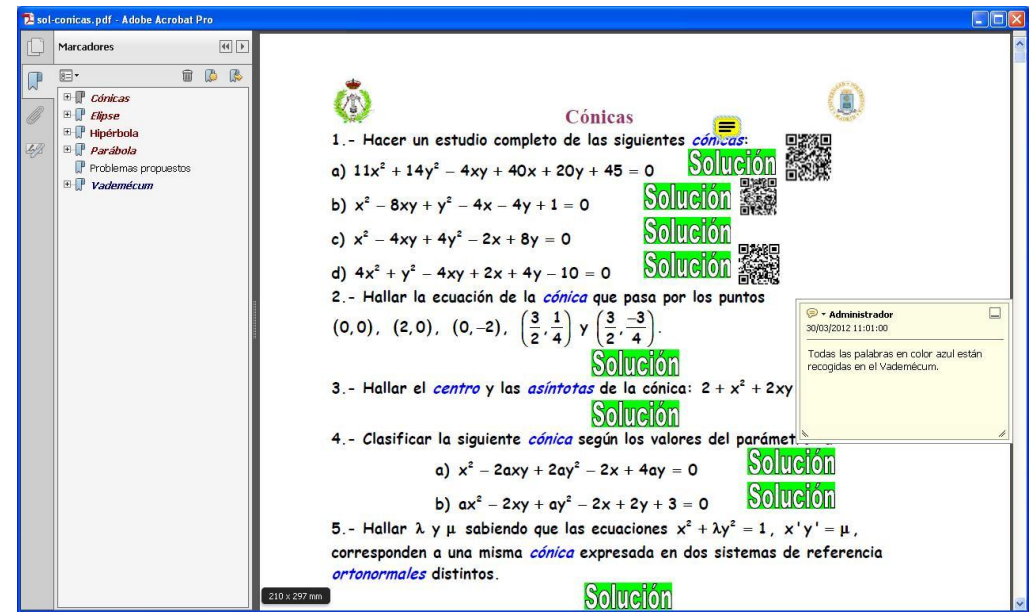

Fig. 5. Archivos de ejercicios resueltos.

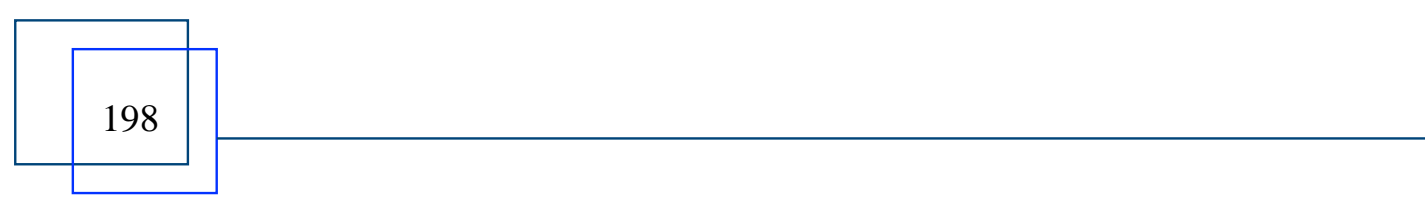




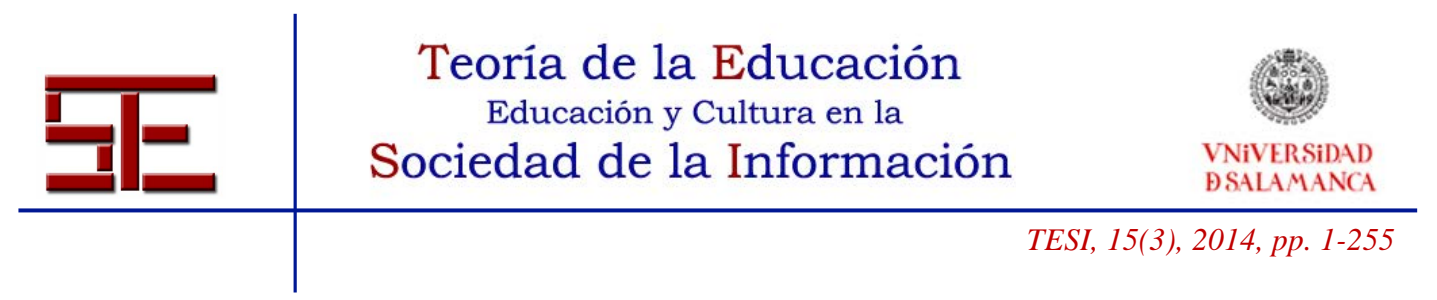

5. Test de autoevaluación: existe un banco de 80 preguntas, apareciendo 20 aleatoriamente seleccionadas de las 80. Cada cuestión ofrece cuatro posibles respuestas, siendo válida solo una de ellas. Una vez seleccionada la opción elegida y pulsado el botón "Submit", el sistema indicará si es correcta o no, proporcionándose una explicación tanto en el caso de que se acierte como en el que se falle (ver Fig. 6).

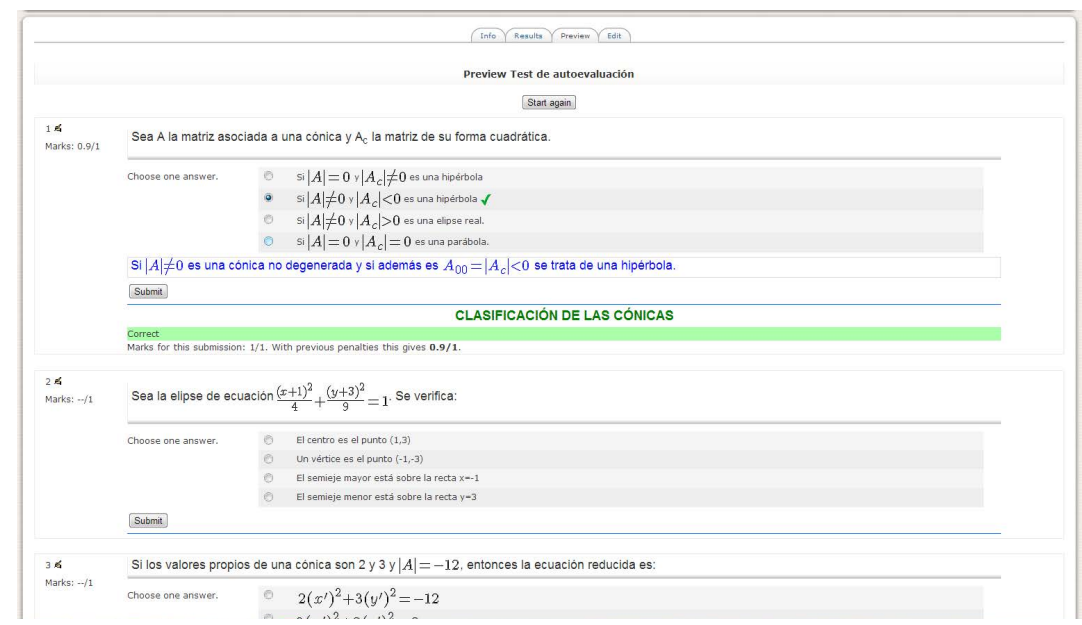

Fig. 6. Test de autoevaluación.

\subsection{Sistemas de acceso al WorkShop}

Merece destacarse que los alumnos pueden conectarse al workshop a través de su teléfono móvil con conexión a internet; estamos ante el m-learning (mobile-learning) que significa literalmente aprendizaje móvil. "Es la integración del e-learnig (sistema de enseñanza y aprendizaje a través de redes digitales) con los dispositivos móviles de comunicación" (García, 2004, 138). El término mobile-learning aparece por primera vez en Estados Unidos a finales de los 90 y se instaura en Europa a inicios del siglo XXI.

En este trabajo, se ha realizado una disponibilidad de todos los recursos en un acceso desde un móvil para facilitar la autonomía y el acceso a la información en cualquier momento y lugar. En particular:

- Los vídeos, además de la opción más favorable para visualizarlos en el ordenador (PC) con el formato Windows Media Player, se han instalado con el formato MP4 de tamaño reducido y específico para móvil. Con la posibilidad de acceder a ellos mediante un código QR (o Bidi) insertado en los documentos

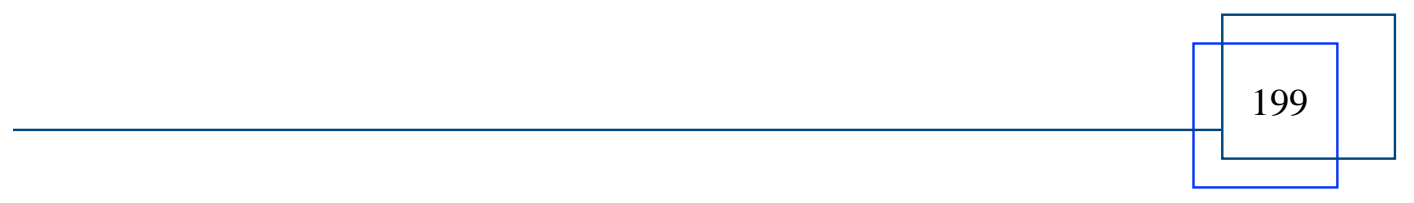


PDF, de tal forma que si se está trabajando con el documento impreso no es necesario nada más que escanear con el móvil para visualizar los contenidos correspondientes (ver Fig.7)

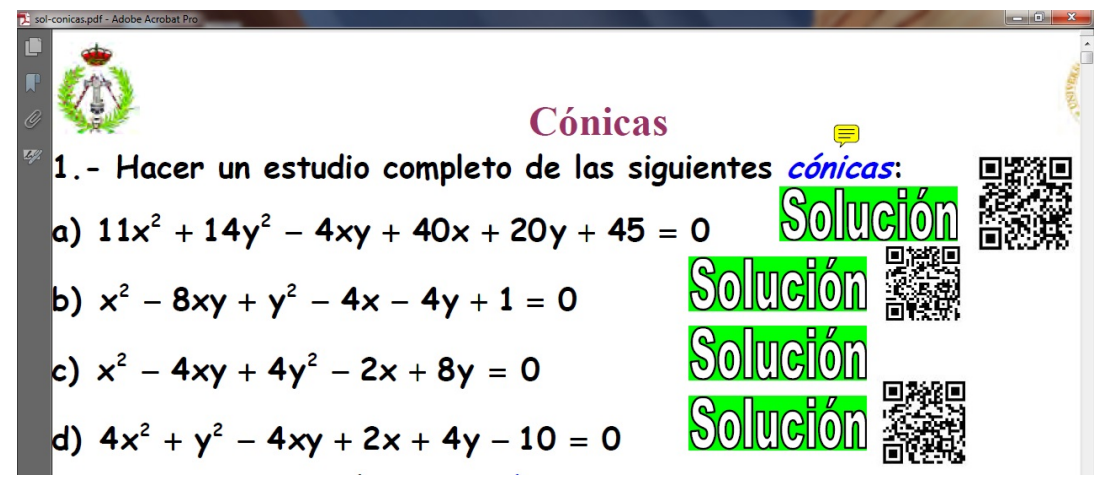

Fig. 7. Visualización.

- El uso de WOLFRAMALPHA, como asistente matemático se puede realizar, igualmente desde un móvil (ver Fig. 8).

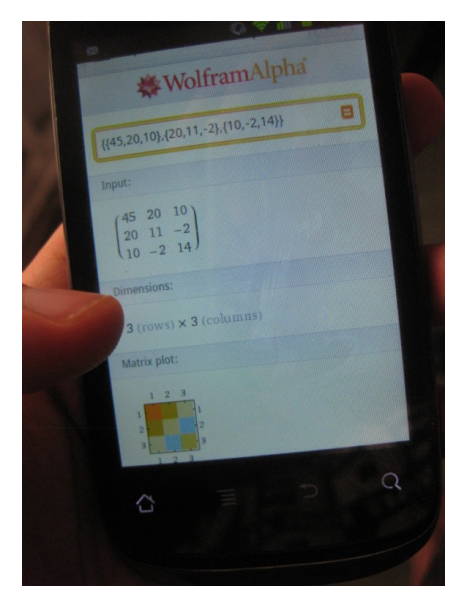

Fig. 8. Wolframalpha en la pantalla de un teléfono móvil.

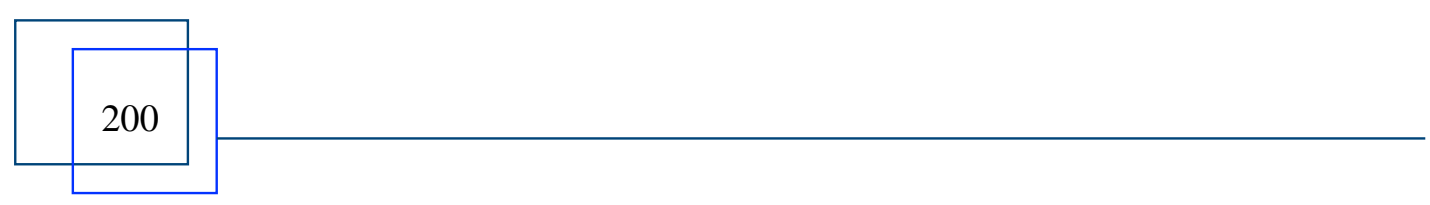




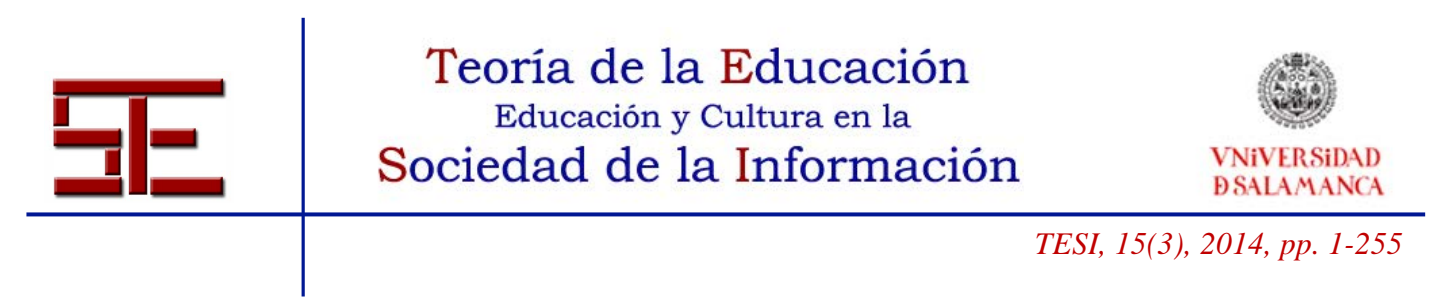

- La plataforma de tele-enseñanza MOODLE es sin duda uno de los gestores de cursos open source más utilizados hoy en día. Se trata de un sistema de gestión de la enseñanza (course management system o learning management system) basado en la teoría constructivista del aprendizaje.

- Las diferentes experiencias llevadas a cabo en este sector, por diferentes autores, han demostrado que el Aprendizaje Móvil es realmente efectivo en:

- Aprendizajes basados en la resolución de problemas y mejora de determinadas habilidades.

- Aprendizajes al aire libre o para trabajos de campo.

- Aprendizajes en instituciones culturales. En estos entornos se ha demostrado que las tecnologías multimedia e inalámbricas son una eficaz herramienta, ya que proporcionan al usuario información de interés en función del lugar en el que se encuentre.

- Reciclaje profesional.

- Por otra parte, los inconvenientes son:

- Pantallas pequeñas de los móviles. Esto conlleva dificultades en la lectura de textos medianos, la cantidad de información visible es limitada y el desplazamiento continuo por la pantalla para leer toda la información.

- Existen pocas aplicaciones educativas.

- Dificultades o imposibilidad de instalar y usar determinado software.

\subsection{Aspectos complementarios}

Los videos constituyen una herramienta educativa ya que pueden ser utilizados para fomentar la comprensión y los aprendizajes. M. Schmidt (1987) distingue tipos diferentes de vídeos en función de los objetivos didácticos. Pueden ser instructivos, su cometido es instruir o lograr que los alumnos dominen un determinado contenido. Cognoscitivos, si su misión es dar a conocer diferentes aspectos relacionados con el tema que están estudiando. Motivadores, para disponer positivamente al alumno hacia el desarrollo de una determinada tarea. Modelizadores, presentan modelos a imitar o a seguir y Lúdicos o expresivos destinados a que los alumnos puedan aprender y comprender el lenguaje de los medios audiovisuales.

Los videos utilizados en este workshop estarían dentro de los vídeos que M. Schimidt clasifica como Instructivos, ya que presentan contenidos que los alumnos deben dominar, Motivadores, porque pretenden motivar al alumno en el estudio de las cónicas,

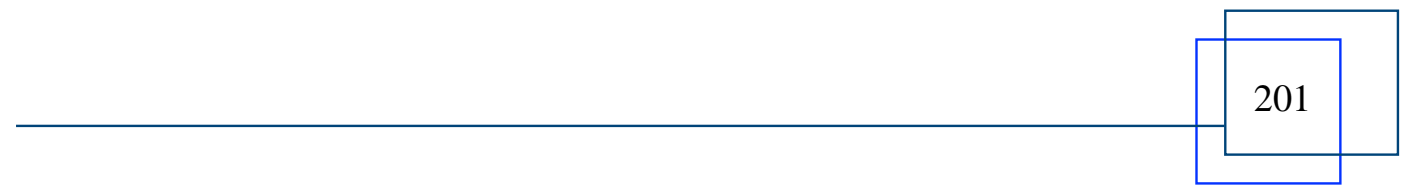




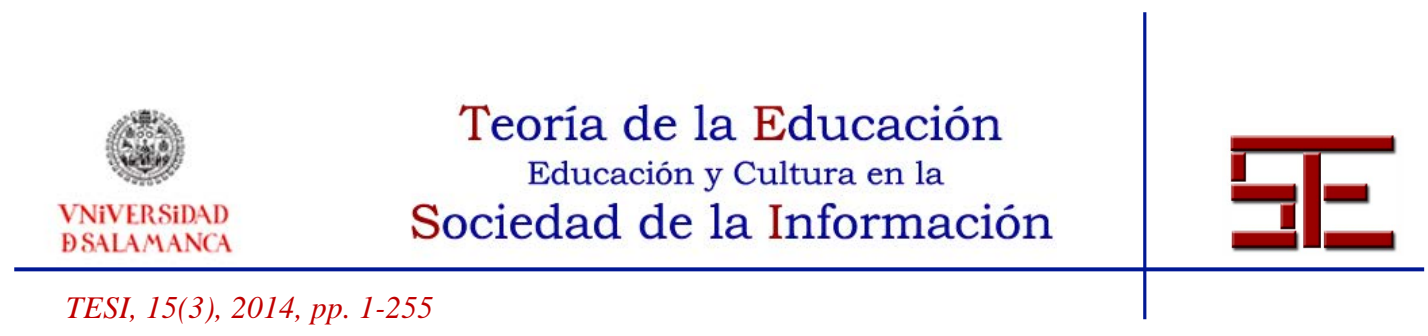

introduciendo los ejercicios con casos reales, y Modelizadores, ya que presentan problemas a imitar o seguir. Para la creación de dichos vídeos ha sido necesario un equipo multidisciplinar formado por profesores y expertos en comunicación audiovisual.

\section{Descripción de la creación de los vídeos}

La UPM dispone del Gabinete de Tele-Educación (GATE) que es un servicio universitario de apoyo para la integración de las tecnologías de la información y las comunicaciones en la enseñanza de la mencionada Universidad.

Las actividades de este Servicio comprenden la gestión de la plataforma de teleenseñanza de la UPM, servicios audiovisuales para las actividades educativas, el servicio de laboratorios virtuales, así como un servicio de orientación técnicopedagógica para la docencia.

Dependiendo del tipo de actividad, los técnicos del GATE se desplazan a los diferentes Centros de la UPM para realizar las grabaciones o son los profesores los que acuden a su sede en el Rectorado para llevar a cabo la grabación. Los vídeos de este Workshop fueron grabados con el apoyo y los medios del GATE en su sede.

El vídeo de presentación del taller está grabado con la técnica de "pantalla verde", para después colocar como fondo la propia imagen de la profesora explicando los diferentes apartados del Workshop que van apareciendo a su derecha mostrando lo que el alumno se irá encontrando a medida que se adentre en el curso (ver Fig. 9).
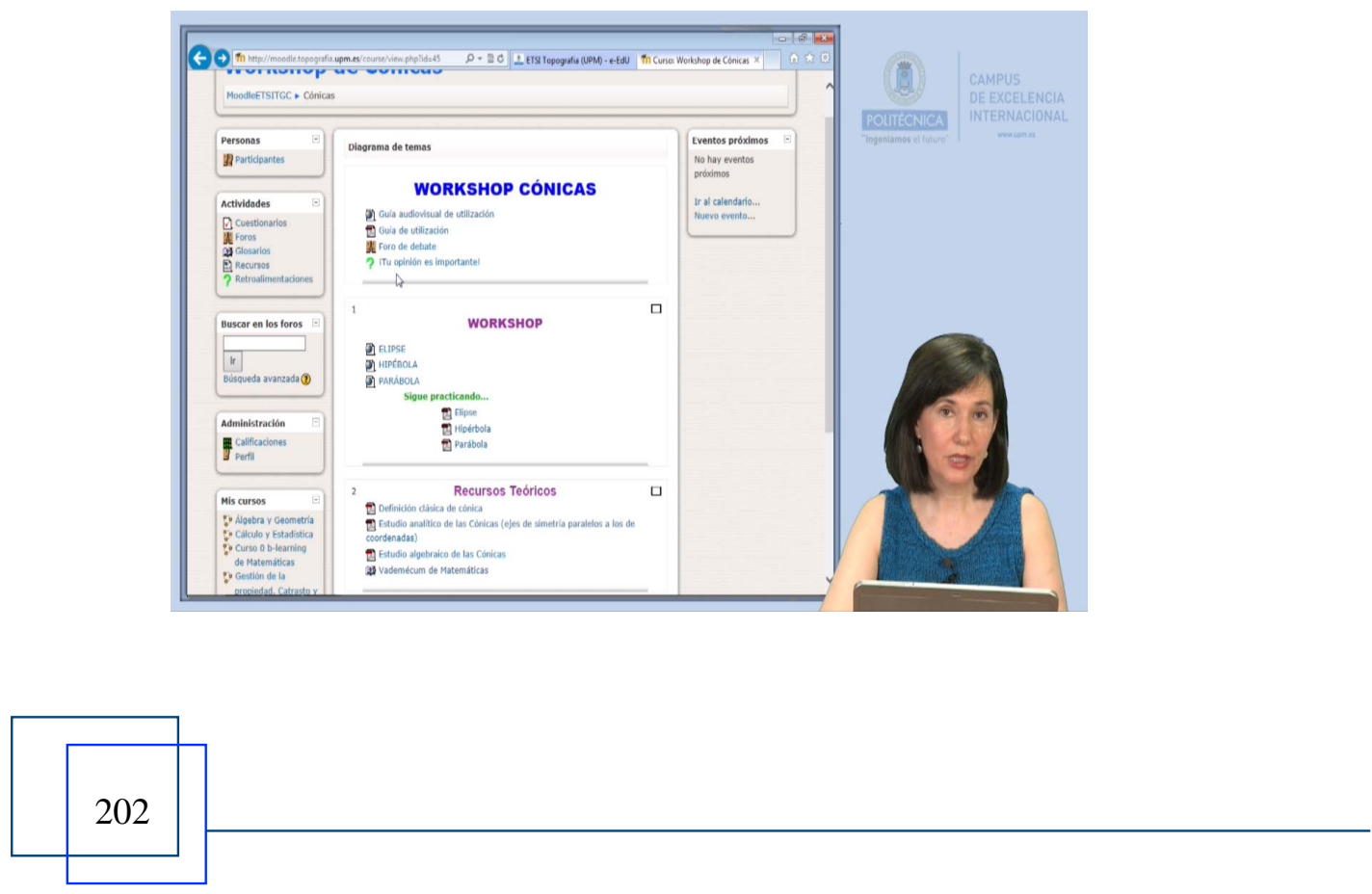


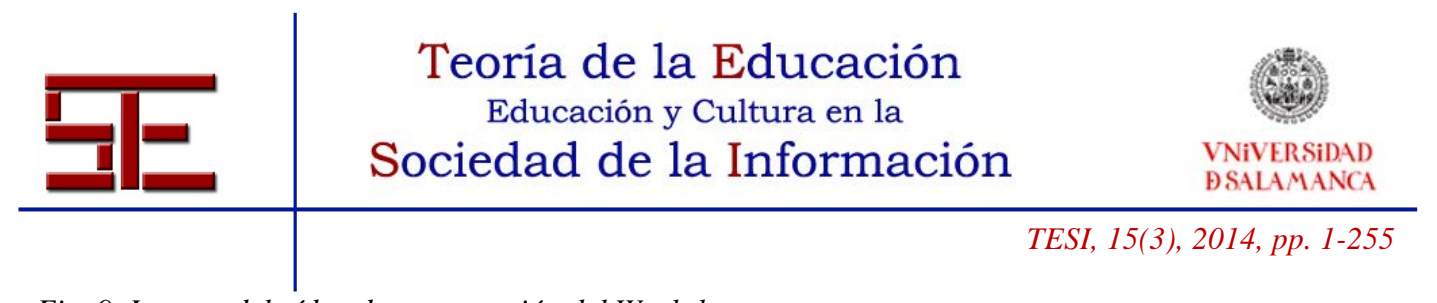

Fig. 9. Imagen del vídeo de presentación del Workshop.

Los vídeos de cada uno de los tres tipos de cónicas recogen paso a paso el planteamiento y los cálculos que se van realizando y que van apareciendo paulatinamente en la pantalla del ordenador, que es la que se muestra en el vídeo, bajo las explicaciones minuciosas de la profesora cuya presencia se adivina por la voz y el puntero que va apareciendo en la pantalla (ver Fig. 10).

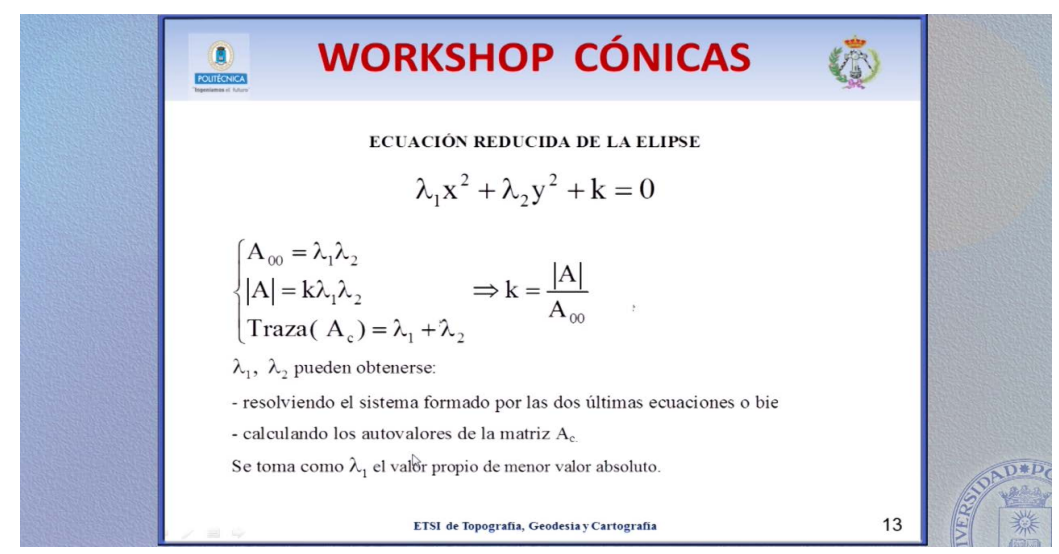

Fig. 10. Imagen del vídeo correspondiente a la elipse.

\section{METODOLOGÍA}

El desarrollo de las sesiones consiste en un recordatorio sobre propiedades métricas de las cónicas vistas en bachillerato que no puede durar más de 15 minutos y a continuación visionado del vídeo correspondiente a una de las tres cónicas objeto de estudio (20 minutos) para realizar el problema propuesto de forma individual o colectiva bajo la supervisión del profesor (85 minutos). Par finalizar el alumno realiza el cuestionario de autoevaluación (20 minutos).

La organización del workshop tiene en cuenta tres tipos de objetivos que asociamos con distintos niveles de dificultad en el aprendizaje.

- El primer tipo de objetivos es el instrumental. Se trata de adquirir entrenamiento en los cálculos: cálculo matricial y determinantes, resolución de ecuaciones, etc.

- El segundo tipo es el de comprensión y aplicación de conceptos, donde a su vez establecemos una escala según el grado de complejidad de los mismos.

- El tercer tipo es el de relacionar los diferentes conceptos, con el fin de ayudar a los alumnos a comprender los elementos característicos de las cónicas.

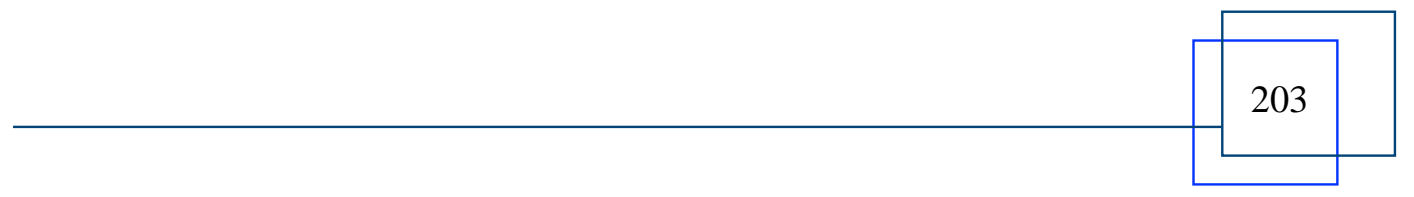




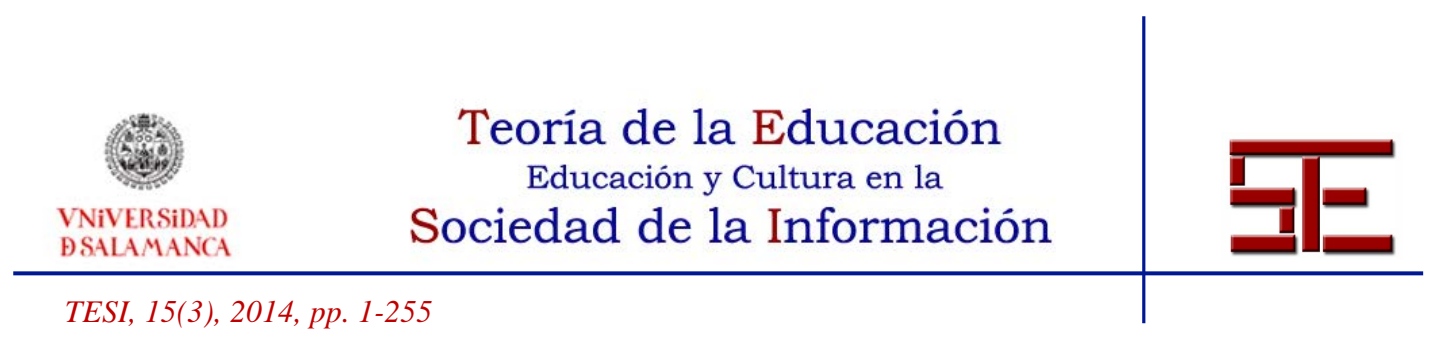

\section{RESULTADOS}

Los resultados observados en los alumnos con los que se ha venido trabajando en la Escuela Técnica Superior de Ingenieros en Topografía, Geodesia y Cartografía, han sido:

- Se ha comparado la curva de aprendizaje de Wolfram con la del programa Derive para los alumnos de primer curso de Grado, obteniéndose unos resultados bastante más favorables en cuanto a tiempo de aprendizaje con Wolfram que con Derive.

- Es destacable que el idioma inglés, en este caso, no ha sido un problema para los estudiantes y este tipo de aplicaciones, disponibles en dispositivos móviles o bien en ordenadores, permite una mayor flexibilidad en la dinámica del aula.

- En cuanto al propio taller, se ha constatado una mayor motivación de los alumnos cuando utilizan este tipo de estudio frente a los métodos tradicionales y el autoaprendizaje es más efectivo.

El resultado del trabajo realizado se compone de un conjunto de contenidos en Moodle (contenidos teóricos, flujos de trabajo, ejercicios resueltos, ejercicios propuestos, test de autoevaluación) entre los que se encuentra un video realizado a medida para este workshop, disponible para todos los alumnos de la UPM, con el objetivo de que nos permita evaluar si hay una motivación hacia el aprendizaje de las cónicas, además de una mejora en los resultados académicos de los alumnos.

Para valorar la satisfacción de los encuestados y mejorar el workshop de cónicas, así como otros talleres ya previstos, se realizó una encuesta a 30 alumnos de la ETS de Ingenieros en Topografía, Geodesia y Cartografía de la UPM. Dicha encuesta se divide en tres bloques de preguntas: 1. Contenido pedagógico y organizativo. 2. Moodle: presentación, diseño y facilidad de uso. 3. Valoración general y comentarios.

Los resultados se presentan en la tabla siguiente:

Tabla 1. Resultados de la encuesta,

\begin{tabular}{lllll}
\hline Resultados de la encuesta & & & & \\
Preguntas & Mucho(4) & Bastante(3) & Poco(2) & Muy Poco(1) \\
Vídeos-Móvil & $48 \%$ & $52 \%$ & $0 \%$ & $0 \%$ \\
Recursos teóricos & $52 \%$ & $32 \%$ & $8 \%$ & $4 \%$ \\
Recursos prácticos & $44 \%$ & $56 \%$ & $0 \%$ & $0 \%$ \\
\hline
\end{tabular}

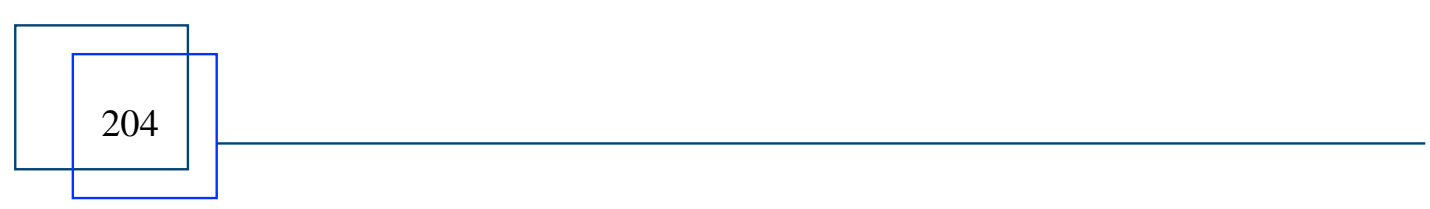




\begin{tabular}{|c|c|c|c|c|}
\hline$\square$ & \multicolumn{3}{|c|}{$\begin{array}{c}\text { Teoría de la Educación } \\
\text { Educación y Cultura en la } \\
\text { Sociedad de la Información }\end{array}$} & $\begin{array}{l}\text { VNiVERSiDAD } \\
\text { DSALAMANCA }\end{array}$ \\
\hline & \multicolumn{4}{|r|}{ TESI, 15(3), 2014, pp. 1-255 } \\
\hline Vademécum & $32 \%$ & $36 \%$ & $16 \%$ & $0 \%$ \\
\hline Autoevaluación & $28 \%$ & $56 \%$ & $4 \%$ & $0 \%$ \\
\hline Foro & $8 \%$ & $20 \%$ & $44 \%$ & $8 \%$ \\
\hline Aspecto gráfico & $20 \%$ & $68 \%$ & $8 \%$ & $0 \%$ \\
\hline Claridad de información & $40 \%$ & $56 \%$ & $0 \%$ & $0 \%$ \\
\hline Cantidad de información & $44 \%$ & $52 \%$ & $0 \%$ & $0 \%$ \\
\hline $\begin{array}{l}\text { Rapidez de descarga de la } \\
\text { página }\end{array}$ & $36 \%$ & $40 \%$ & $20 \%$ & $4 \%$ \\
\hline Facilidad de uso & $44 \%$ & $44 \%$ & $12 \%$ & $0 \%$ \\
\hline $\begin{array}{lll}\text { ¿Estás satisfecho con } & \text { el } \\
\text { aprendizaje obtenido en } & \text { el } \\
\text { WorkShop Cónicas? } & & \end{array}$ & $32 \%$ & $68 \%$ & $0 \%$ & $0 \%$ \\
\hline $\begin{array}{l}\text { ¿Recomendarías este Work- } \\
\text { Shop Cónicas a un amigo? }\end{array}$ & SI $100 \%$ & No & $0 \%$ & \\
\hline
\end{tabular}

A continuación, exponemos algunos comentarios realizados por los alumnos en dicha encuesta:

- "Me parece muy bien este tipo de enseñanza, tendríais que ampliar a más temas y no solo en cálculo sino en todas las asignaturas (sobre todo física)".

- "Intentar hacer videos de todos los temas que se dan en las asignaturas (calculo, algebra....".

- "Seguir adelante, mola mucho".

- "Se agradece el trabajo realizado por los profesores, porque en mi opinión todo esto es una herramienta muy valiosa para los alumnos. Nos ayuda a comprender mejor y a practicar la materia".

- " $m m$ de momento nada".

Esta apreciación positiva de los alumnos se encuentra en consonancia con la tesis expresada por C. Alonso y D. Gallego (1999) en el libro "Multimedia en la Web", según la cual los alumnos retienen la información de la siguiente manera:

- El $10 \%$ de lo que leen.

- EL 20\% de lo que escuchan.

- El $30 \%$ de lo que ven.

- El 50\% de lo que ven y escuchan. 


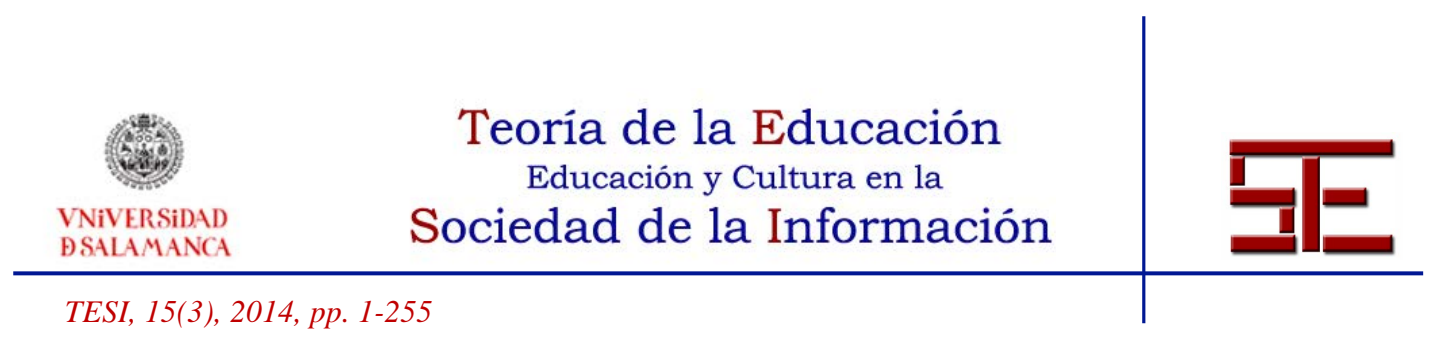

- El 70\% de lo que se dice y se discute.

- El 90\% de lo que se dice y luego se realiza.

Con la educación- $m$, y las tecnologías empleadas en este workshop nos acercaríamos a los porcentajes finales de dicha clasificación. Creemos que estaríamos en la pedagogía informal, ya que se ofrece la adquisición del conocimiento en un esquema más libre. Esto no significa que carece de control, sino más bien que está incrustado, tanto en el espacio como en las situaciones particulares a las que se enfrenta el alumno.

Se ha observado una mayor motivación de los estudiantes debido a los vídeos explicativos. Los propios alumnos demandan a los profesores vídeos de otros temas de la asignatura tras utilizar los del workshop.

Un aspecto a mejorar es la comunicación de los alumnos entre sí a través del foro y conseguir que realicen un mayor número de tutorías.

Además, cabe señalar que esta metodología es aplicable a las ciencias básicas (física, química, biología, etc.), por tanto, lo presentamos como metodología transferible a otras áreas tal y como lo solicitan los alumnos encuestados. Concretamente nos estamos refiriendo a grabaciones de vídeos explicativos tanto teóricos como prácticos, problemas resueltos paso a paso, descripción de ejemplos teóricos, etc. Resulta una metodología adaptable al criterio de cada profesor y de los objetivos a conseguir en cada situación.

\section{CONCLUSIONES}

Este tipo de Workshops proporciona una atención más personalizada, permitiendo dar respuesta a los distintos ritmos de aprendizaje y fomentar la adquisición del conocimiento sobre las cónicas. En concreto, este taller permite que alumnos de nivel universitario obtengan una serie de conocimientos teóricos acerca de las secciones cónicas y que dominen un flujo de trabajo completo para la clasificación y obtención de sus elementos.

Las herramientas de cálculo de Wolfram y las posibilidades de representación gráfica permiten ir desentrañando "paso a paso" y de forma progresiva, cada uno de los conceptos que se encuentran bajo los elementos de las cónicas.

La disponibilidad del programa Wolfram para varios dispositivos supone ser independiente de programas que necesitarían su instalación y posiblemente un coste adicional.

El uso de dispositivos móviles ha permitido una mayor libertad y flexibilidad en el aprendizaje al no depender del espacio físico del aula y estar sujeto a un horario

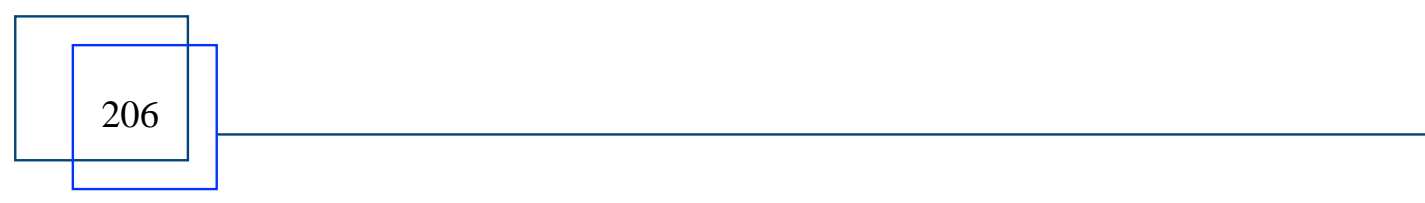




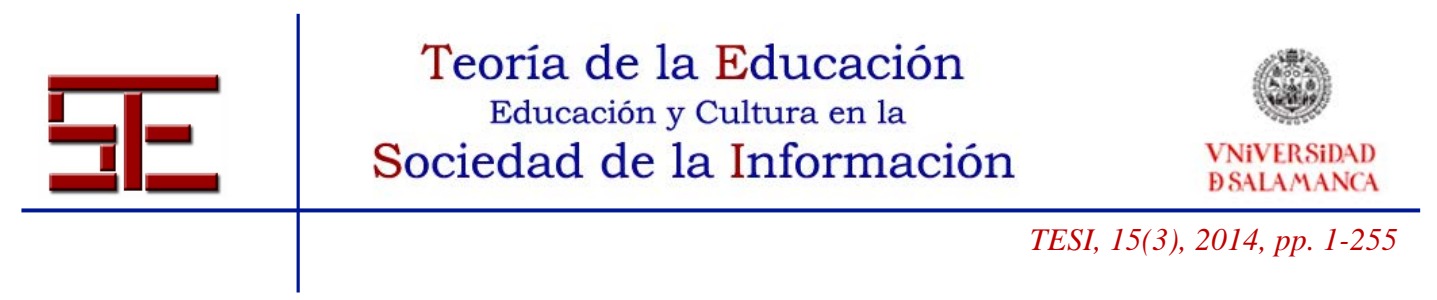

determinado. Además de la interacción instantánea entre alumno-profesor, con dudas resueltas casi de forma inmediata.

Este workshop de cónicas, es el resultado de la labor de un grupo de profesores dedicados a la innovación educativa durante varios años. Cada curso académico mejora al anterior y el trabajo que presentamos es el más actualizado.

Especialmente cabe señalar que esta metodología es aplicable a las ciencias básicas (física, química, biología, etc.), por tanto, la consideramos como una metodología transferible a otras áreas, particularmente a grabaciones de vídeos explicativos, tanto teóricos como prácticos, problemas resueltos paso a paso, descripción de ejemplos teóricos, etc.

Por último, queremos remarcar la gran satisfacción que nos produce el hecho de percibir que nuestros alumnos nos agradecen el esfuerzo que realizamos para mejorar el aprendizaje mediante las TIC.

También, señalar que esta forma de trabajar, fue galardonada con el primer premio de la IV edición de los Premios MEC-Universia dentro de la Iniciativa en OCW 2010. Por último, indicar la que está siendo nuestra aportación en la difusión en la enseñanza de las matemáticas mediante las TIC:

1. Colaborar para la aplicación de todas las tecnologías que repercuten en la generación, difusión y utilización del conocimiento.

2. Ayudar a alumnos y profesores, mediante el aprendizaje invisible, para que el saber matemático sea más sencillo y atractivo (Morillo M. C, Sebastián L., Casado M. L., 2011).

\section{BIBLIOGRAFÍA}

Aguaded, I. (2010). Aprender y enseñar con las tecnologías de la comunicación. Rev. Agora digital. Disponible en: $<$ http://www.uhu.es/agora/version01/digital/numeros/01/01articulos/monografico/aguaded.pdf $>$.

ASINCAP Móvil learning, en: $<$ http://www.asincap.cl/index.php?option=com_content\&view=article\&id=101\&Itemid $=103>$.

Ausbel, D.P. (1976). Psicología educativa: un punto de vista cognoscitivo. (1 $\left.{ }^{\mathrm{a} e d}\right)$ México: Trillas. 


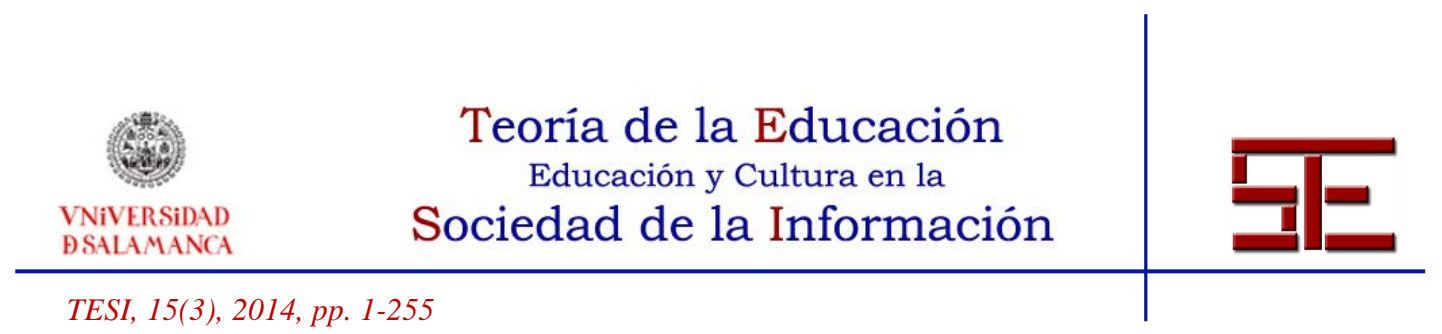

Barrows, H.S. (1986). A Taxonomy of problem-based learning methods, en Medical Education. 20(6), 481-486.

$<$ Blog.educalab.es/cniie>.

Brazuelo Grund, F. y Gallego Gil, D. J. (2011). Mobile learning. Los dispositivos móviles como recurso educativo. Sevilla: Ed. MAD, SL.

Cabero, J. et al. (2000). Nuevas Tecnologías Aplicadas a la Educación. Madrid: Síntesis.

Gallego, D. y Alonso, C. (1999). Multimedia en la Web. Madrid: Dykinson.

García, L. (2004). Aprendizaje móvil, m- learning. Ed. Bened.

Guzmán, M. (1996). El Rincón de la Pizarra. Madrid: Pirámide

Macias, D. (2007). Las nuevas tecnologías y el aprendizaje de las matemáticas. Revista Iberoamericana de Educación, 42(4).

Morillo M.C, Sebastian L. y Casado M. L. (2011). Diseño para el conocimiento reglado de acceso libre a través de Internet. Revista Arbor, Vol. 187, No Extra 3.

Prieto, L. (2006). Aprendizaje activo en el aula universitaria: el caso del aprendizaje basado en problemas, en Miscelánea Comillas. Revista de Ciencias Humanas y Sociales, Vol. 64., Núm.124, 173-196.

Schmidt, M. (1987). Cine y video educativo. Madrid: MEC.

Synge, J. L. y Griffith, B. A. (1959). Principles of mechanics. New York: McGraw-Hill.

Vagn, L. H. (2001). Traducción de Geometría eterna por Hernández, V. y Villalba, M. PMME-UNISON.

Wikilibros contributors (2009). Aprendizaje combinado/Elementos básicos. Wikilibros.

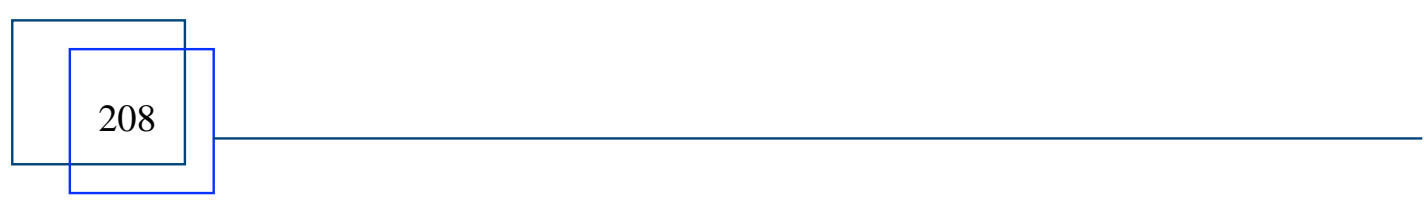

